\title{
Comparison between GVF snake and ED snake in segmenting microcalcifications
}

\begin{abstract}
Snake, active contour or deformable active contour has been widely used in medical image segmentation area. In this paper, comparison between Gradient Vector Flow (GVF) snake and Enhanced Distance (ED) snake in segmenting microcalcifications is carried out. The performance is measured based on actual area of the average percentage difference traced by expert radiologists. Results obtained shows that the values of average percentage difference for the GVF and ED snake are $4.3 \%$ and $6.68 \%$ respectively. These results indicate that the GVF snake has better performance with $95.7 \%$.
\end{abstract}

Keyword: Enhanced distance snake; Gradient vector flow snake; Mammogram; Microcalcifications; Segmentation 